\title{
A secreted splice variant of the Xenopus frizzled-4 receptor is a biphasic modulator of Wnt signalling
}

\author{
Anne-Kathrin Gorny ${ }^{1 \dagger}$, Lilian T Kaufmann ${ }^{1 \dagger}$, Rajeeb K Swain ${ }^{2}$ and Herbert Steinbeisser ${ }^{1 *}$
}

\begin{abstract}
Background: Activation of the Wnt signalling cascade is primarily based on the interplay between Wnt ligands, their receptors and extracellular modulators. One prominent family of extracellular modulators is represented by the SFRP (secreted Frizzled-related protein) family. These proteins have significant similarity to the extracellular domain of Frizzled receptors, suggesting that they bind Wnt ligands and inhibit signalling. The SFRP-type protein Fz4-v1, a splice variant of the Frizzled-4 receptor found in humans and Xenopus, was shown to augment Wnt/B-catenin signalling, and also interacts with those Wnt ligands that act on $\beta$-catenin-independent Wnt pathways.

Findings: Here we show that Xenopus Fz4-v1 can activate and inhibit the $\beta$-catenin-dependent Wnt pathway. Gain-of-function experiments revealed that high Wnt/ $\beta$-catenin activity is inhibited by low and high concentrations of Fz4-v1. In contrast, signals generated by low amounts of Wnt ligands were enhanced by low concentrations of Fz4-v1 but were repressed by high concentrations. This biphasic activity of Fz4-v1 was not observed in non-canonical Wnt signalling. Fz4-v1 enhanced $\beta$-catenin-independent Wnt signalling triggered by either low or high doses of Wnt11. Antisense morpholino-mediated knock-down experiments demonstrated that in early Xenopus embryos Fz4-v1 is required for the migration of cranial neural crest cells and for the development of the dorsal fin.

Conclusions: For the first time, we show that a splice variant of the Frizzled-4 receptor modulates Wnt signalling in a dose-dependent, biphasic manner. These results also demonstrate that the cystein-rich domain (CRD), which is shared by Fz4-v1 and SFRPs, is sufficient for the biphasic activity of these secreted Wnt modulators.
\end{abstract}

Keywords: Fz4-v1, Splice variant frizzled-4 receptor, Biphasic, Wnt signalling, SFRP, Xenopus, Dorsal fin, Neural crest

\section{Findings}

Fz4-v1 demonstrates a dose-dependent, biphasic activity in modulating $\mathrm{Wnt} / \beta$-catenin-dependent signalling SFRPs consist of two domains, an N-terminal cysteinerich domain (CRD) and a C-terminal Netrin-like domain [1-3]. Based on similarity with the CRD region of Frizzled receptors, SFRPs were originally described as classical Wnt antagonists [4], however, recent data also describe agonistic functions of SFRPs [5-9]. These gainof-function experiments in cultured cells and Xenopus embryos demonstrated that low levels of SFRPs enhance the $\mathrm{Wnt} / \beta$-catenin pathway, but high levels of SFRPs suppress Wnt signalling $[7,8]$.

\footnotetext{
* Correspondence: Herbert.Steinbeisser@med.uni-heidelberg.de

${ }^{\dagger}$ Equal contributors

${ }^{1}$ Section Developmental Genetics, Institute of Human Genetics, University of Heidelberg, Im Neuenheimer Feld 366, Heidelberg D-69120, Germany
}

Full list of author information is available at the end of the article
Xenopus Fz4-v1 is an SFRP-type protein which is generated from the Frizzled-4 receptor mRNA by intron retention. In contrast to classical SFRPs Fz4-v1 includes a CRD domain but lacks a Netrin-like domain [9]. Such splice variants have been described for both the human and Xenopus Frizzled-4 receptor and were previously named Fz4S [5,9]. Xenopus Fz4-v1 is a secreted protein and can modulate $\mathrm{Wnt} / \beta$-catenin signalling in a non-cell autonomous manner (Additional file 1: Figure S1).

Both human and Хеnopus Fz4 splice variants were shown to enhance the activity of Wnt ligands, which activate the Wnt/ $\beta$-catenin pathway [5,9]. Xenopus Fz4-v1 also interacts with Wnt ligands of the Wnt5a class, but its effect on $\beta$-catenin-independent Wnt signalling has not been assessed [9]. Because previous experiments had only demonstrated an activating function of Fz4-v1, we tested whether Fz4-v1 might also have an inhibitory activity on Wnt/ $\beta$-catenin signalling.

\section{Ciomed Central}


To investigate the dual function of Fz4-v1, we took advantage of the Xenopus axis duplication assay, which provides a sensitive and reliable system to test Wnt activities (Figure 1A-F). Injection of 10 pg wnt8b RNA into the ventral marginal zone caused ectopic axis formation in more than $70 \%$ of the injected embryos. However, co-injection of 250 pg fz4-v1 RNA completely blocked the formation of secondary body axes. When low doses of wnt8b ( $0.5 \mathrm{pg})$ were injected, which alone were not sufficient to induce a secondary axis, coinjection of $1 \mathrm{ng} f z 4-v 1$ induced ectopic axes in more than $50 \%$ of the embryos, consistent with previous studies [5].

In addition, we performed Topflash-Luciferase reporter experiments in Xenopus embryos in order to quantitatively measure Wnt/ $\beta$-catenin signalling strength (Figure 1G, $\mathrm{H})$. Xenopus embryos were injected with synthetic mRNAs for wnt3a, fz4-v1 and the Topflash-Luciferase reporter plasmid. Wnt activity was measured at gastrula stage. Fz4v1 alone did not activate the Topflash-Luciferase reporter. However, Luciferase activity generated by low doses of wnt3a ( $0.05 \mathrm{pg}$ and $0.1 \mathrm{pg}$ ) was enhanced by co-injection of 5 and $50 \mathrm{pg}$ of $f z 4-v 1$, but repressed by $1000 \mathrm{pg}$. In contrast, strong Wnt/ $\beta$-catenin signals triggered by high doses of $w n t 3 a(5 \mathrm{pg})$ were inhibited by both, high (1000 pg) and low (5 and $50 \mathrm{pg}$ ) amounts of $f z 4-v 1$ (Figure $1 \mathrm{H}$ ).

This analysis revealed that Fz4-v1 can act as a biphasic modulator of Wnt/ $\beta$-catenin signalling. Therefore Fz4-v1 behaves like classical SFRPs, which activate Wnt signalling at low and inhibit at high doses $[7,8]$. The presence of the CRD domain is sufficient to induce biphasic activity, because Fz4-v1 is lacking the NTR domain. Recent structural analysis of Wnt8 interaction with the CRD domain of Fz8 could explain the finding that low doses of SFRPs activate, but high doses inhibit the activity of Wnt ligands [10]. Low CRD concentration could weaken the attachment of the lipid modified Wnt proteins to the plasma membrane and ECM. Wnts would become more diffusible and association with the receptors would be facilitated. High concentration of CRD could induce the clustering of Wnt/CRD complexes, rendering them inactive. Since SFRPs can bind to Frizzled receptors, it would also be plausible that high concentrations of secreted CRD domains could cause receptor silencing.

In case of strong Wnt/ $\beta$-catenin signals induced by high concentration of Wnt ligands one can assume that all the endogenous Wnt receptors are occupied. In this case the CRD domains could compete with Wnt ligands
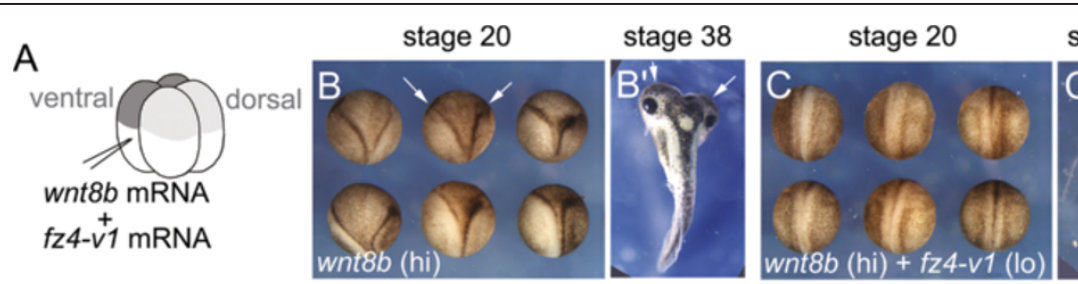

stage 38
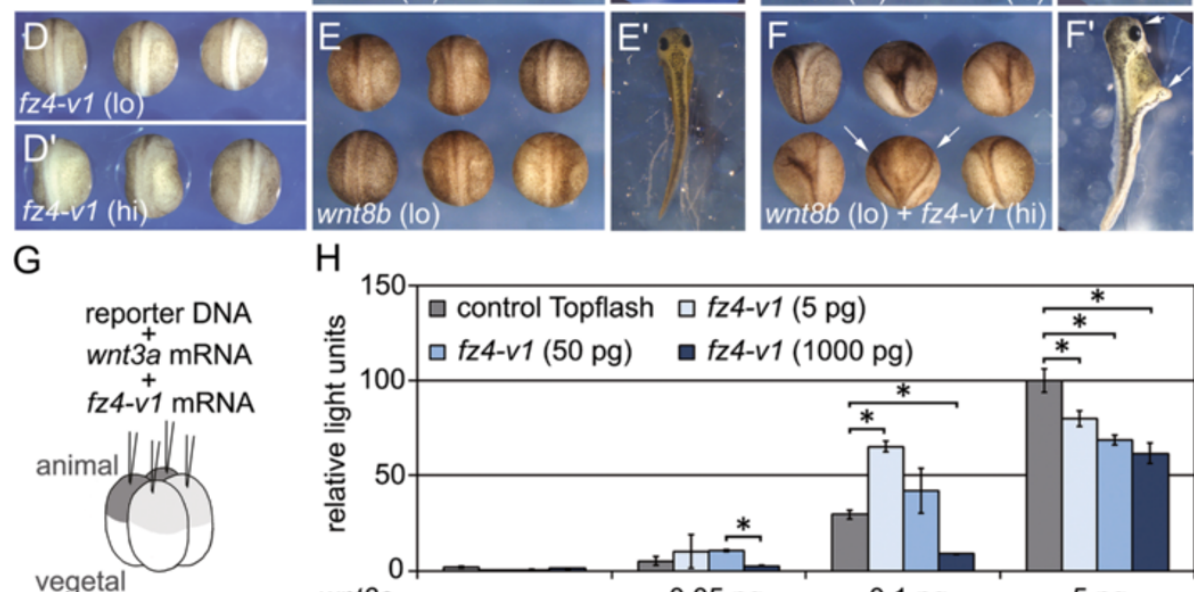

$\mathrm{H}$

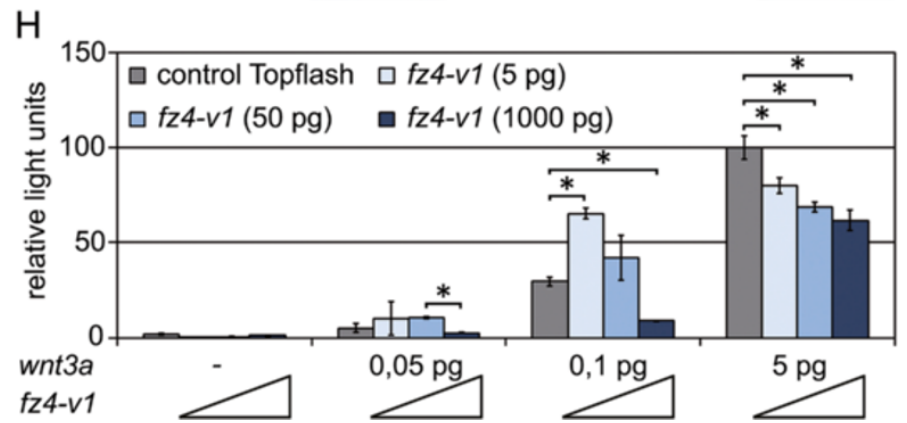

Figure 1 Fz4-v1 has a biphasic, dose-dependent activity in modulating Wnt/B-catenin-dependent signalling. (A-F) 4-cell stage embryos were injected on the ventral marginal side with $0.5 \mathrm{pg}$ wnt8b RNA (lo) or $10 \mathrm{pg}$ (hi) alone, or in combination with 250 pg fz4-v1 RNA (lo) or 1000 pg (hi) or with 250 pg (lo) or 1000 pg (hi) fz4-v1 RNA alone. Formation of secondary body axes was scored at neurula (st. 20, B-F) and tadpole stages (st. 38, $\left.\mathbf{B}^{\prime}-\mathbf{F}^{\prime}\right)$. Frequency of secondary axis formation: (B, $\left.\mathbf{B}^{\prime}\right) 71.9 \%(n=36),\left(\mathbf{C}, \mathbf{C}^{\prime}\right) 0 \%(n=24),\left(\mathbf{D}^{\prime}, \mathbf{D}^{\prime}\right) \mathbf{0 \%}(\mathrm{n}=20),\left(\mathbf{E}, \mathbf{E}^{\prime}\right) \mathbf{0 \%}$ $(n=24),\left(\mathbf{F}, \mathbf{F}^{\prime}\right) 54.2 \%(n=24)$. (G) At the 4-cell stage blastomeres were injected in the animal region with $80 \mathrm{pg}$ Topflash-Luciferase reporter plasmid in combination with wnt3a RNA $(0.05 \mathrm{pg}, 0.1 \mathrm{pg}, 5 \mathrm{pg})$ and fz4-v1 RNA (5 pg, 50 pg, $1000 \mathrm{pg})$. (H) Luciferase activity was measured at gastrula stage (st. 11). Error bars represent standard deviation (SD). (*) indicates significant difference (Student's $t$ test, $p<0.05$ ). 
for the receptors, which would result in reduced Wnt/ßcatenin activity.

\section{Fz4-v1 activates the Wnt/ $\beta$-catenin-independent JNK pathway}

Wnt5a-type ligands such as Wnt5a and Wnt11 regulate $\beta$-catenin-independent signalling. In Xenopus embryos they orchestrate morphogenetic cell movements by regulating planar cell polarity (PCP) in the mesoderm and the neuroectoderm. Since Fz4-v1 can interact with Wnt5a and Wnt11, we hypothesized that it could interfere with noncanonical Wnt signalling [9]. Overexpression of Fz4-v1 in Xenopus embryos caused malformations such as shortened body axes and spina bifida, indicating that $\beta$-cateninindependent Wnt signalling was perturbed (Figure 2A, B). Since these phenotypes can be generated by either hyper-activation or inhibition of the PCP pathway, it was important to determine whether Fz4-v1 acts as activator or inhibitor.

PCP signalling can be measured using an ATFLuciferase reporter [11], which we made use to analyze the effects of Fz4-v1 (Figure 2C). ATF reporter, wnt11 and fz4-v1 RNAs were injected animally into the two ventral blastomeres of Xenopus embryos at the 4-cell stage, and Luciferase activity was analyzed at gastrula stage. Low doses of wnt11 were only able to induce ATF reporter activity when combined with high amounts of $f z 4-v 1$. ATF reporter activity induced by high concentrations of wht11 was further enhanced by co-injection of fz4-v1 (Figure 2C). The ATF reporter experiment indicated that Fz4-v1 augments Wnt11-mediated PCP signalling. This was confirmed in Xenopus animal cap experiments (Figure 2D-G). Ectodermal animal cap explants stimulated by BVg1, a TGF- $\beta$ growth factor [12], elongate due to convergent-extension (CE) movements that are controlled by the PCP pathway. Explants injected with RNA coding for BVg1 elongated as expected, but co-expression of Fz4-v1 inhibited CE movements. JNK is a downstream effector of the PCP pathway, and coexpression of a dominant negative form of Xenopus JNK1, JNK-APF, antagonized the inhibitory effect of Fz4-v1 in the explants (Figure 2D-G). This demonstrates that Fz4-v1 inhibits CE movements by hyper-activation of PCP signalling and confirms the results of the ATF reporter assay.

\section{In vivo function of Fz4-v1}

To analyze the endogenous function of Fz4-v1 during Хеnopus embryogenesis we performed antisense morpholino knock-down experiments using a translation-blocking morpholino that targets the $5^{\prime}$-UTR. The efficiency and specificity of the morpholino is shown in the Supplements (Additional file 2: Figure S2). Since Fz4-v1 is generated by intron retention, the antisense morpholino also blocks translation of $f z 4$ mRNA. Injection of the Fz4/Fz4-v1
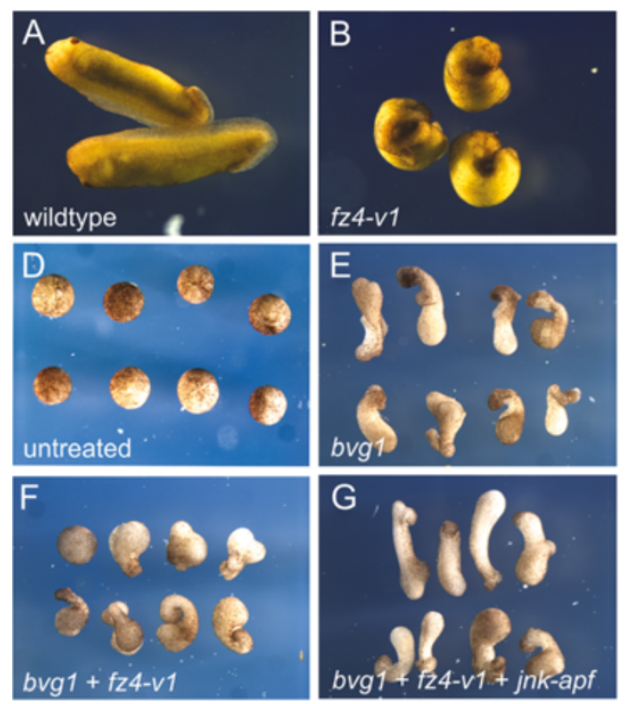

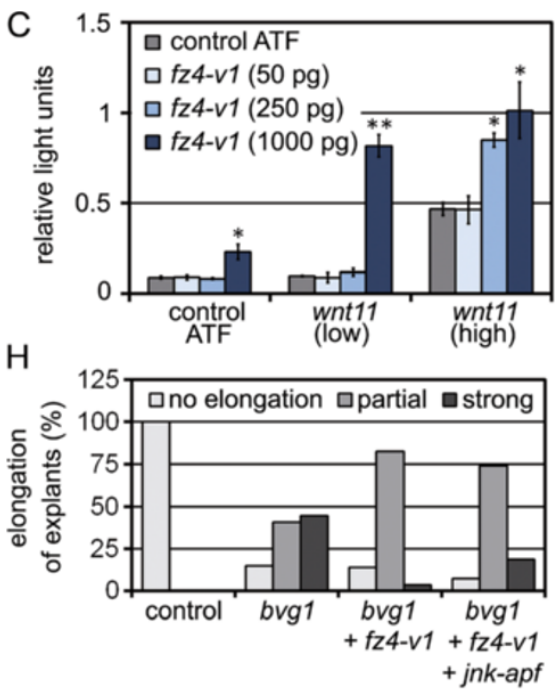

Figure 2 Fz4-v1 perturbs morphogentic movements by activation of the Wnt/B-catenin-independent JNK pathway. (A, B) Embryos were injected radially at the 4-cell stage with $1 \mathrm{ng}$ fz4-v1 RNA. At tailbud stages (st. 25) 22\% displayed spina bifida and neural tube closure defects $(n=45)$. (C) At the 4-cell stage $50 \mathrm{pg}$ of the ATF-luciferase reporter plasmid was injected ventral-animally alone, or in combination with $100 \mathrm{pg}$ (low) or 500 pg (high) wnt11 RNA and fz4-v1 RNA (5,50 and 1000 pg). Reporter activity was analyzed at late gastrula stages (st. 12). Error bars represent SD. $\left(^{*}\right)$ indicate significant difference to control or wnt11 alone, respectively (grey bars) (Student's $t$ test, ${ }^{*} p<0.05$, $\left.{ }^{* *} p<0.001\right)$. (D-H) 4-cell stage embryos were injected at the animal pole region with bvg1 RNA (100 pg) alone, or in combination with fz4-v1 (600 pg) and jnk-apf (1 ng). At blastula stages (st. 9) the caps were explanted and elongation was scored at stage 25. (D) Non-injected controls $(n=20)$ showed no elongation but bvg1-injected explants $(\mathbf{E})$ did $(n=27)$. (F) Co-injection of fz4-v1 partially inhibited explant elongation $(n=29)$, which was restored by JNK-APF $(n=27)$ (G). (H) Quantification of the animal cap experiments. 
morpholino at the 2-or 4-cell stage had no adverse effect on early embryogenesis, suggesting that Fz4-v1 and Fz4 do not affect maternal and early zygotic Wnt signalling, since such interference are expected to result in dorsoventral patterning defects.

At tailbud stages, however, Fz4/Fz4-v1-depleted embryos displayed defective dorsal fin development (Figure 3A-E). When the antisense morpholino was injected only in one side of the embryo in situ hybridization for sox 10 and twist mRNA revealed that migration of cranial neural crest (CNC) cells was inhibited (Figure 3F-G). Both, the dorsal fin phenotype and the $\mathrm{CNC}$ migration defect could be specifically rescued by co-injection of $f z 4-v 1$ RNA. Since Fz4-v1 can rescue the morpholino effect despite its inability to generate a Wnt-dependent Fz4 signal, one can conclude that the morpholino phenotypes are not caused by the knock- down of the Fz4 receptor. Rescue experiments with the full length Fz4 receptor were much less effective (Figure 3D, D'), but Western blot analysis showed that the amount of Fz4-v1 protein was approximately four times higher than that of the full length Fz4 receptor (Additional file 2: Figure S2C). Increasing the amount of $f z 4$ four times to $2000 \mathrm{pg}$, however, resulted in severe malformations of the embryos (Additional file 2: Figure S2E, I). From these experiments we conclude that Fz4-v1 functions in vivo as a modulator of CNC migration and dorsal fin development. Classical experiments demonstrated a contribution of trunk neural crest cells to the mesenchyme in the core of the dorsal fin [13]. In addition, recent data also report an involvement of somites in dorsal fin development [14]. Notably, $f z 4-v 1$ is expressed in the cranial and trunk neural crest, as well as the somitic mesoderm at neurula to tailbud stages
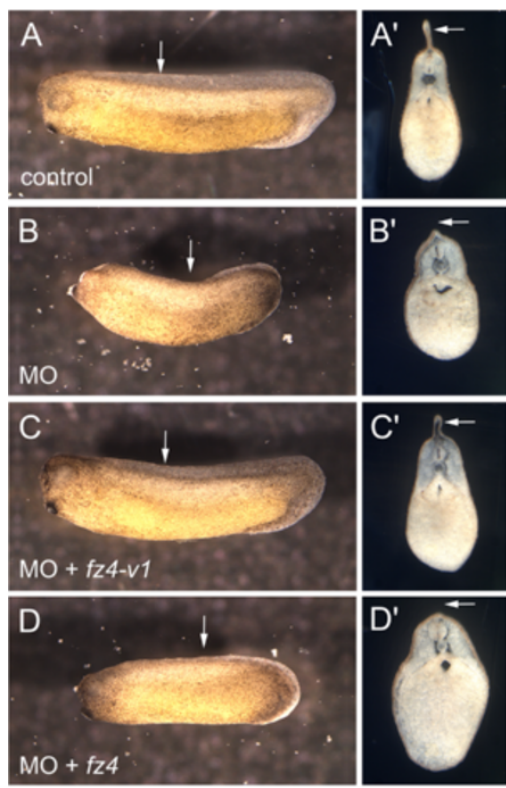

E

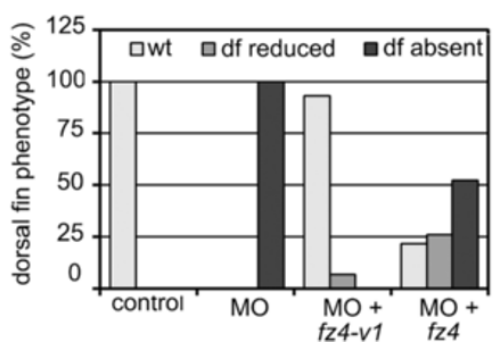

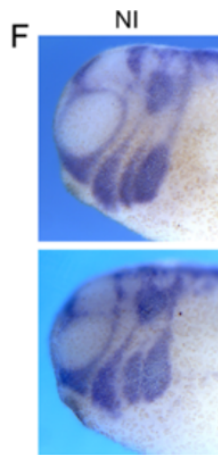
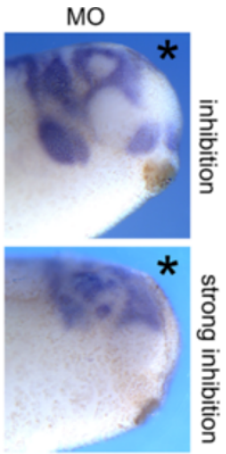

NI

$\mathrm{MO}+f \mathrm{z} 4-\mathrm{v} 1$

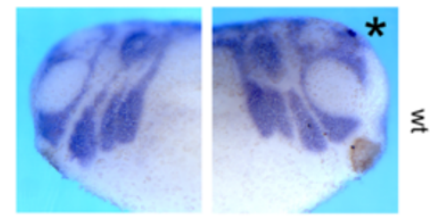

G

$\square$ wt $\square$ inhibition $\square$ strong inhibition

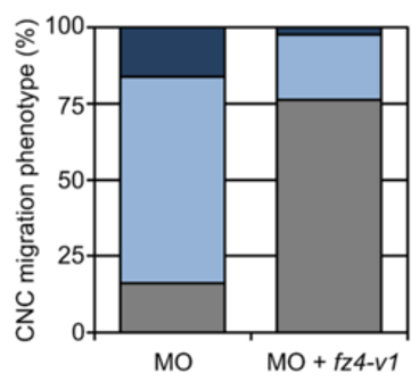

Figure 3 Fz4-v1 function is required for dorsal fin development. (A-D) Embryos were injected at the 2- to 4-cell stage with 50 ng antisense Fz4/Fz4-v1 morpholino oligonucleotide (MO) alone (B, $\left.\mathbf{B}^{\prime}\right)(n=10)$, or in combination with 500 pg fz4-v1 RNA $\left(\mathbf{C}, \mathbf{C}^{\prime}\right)(n=29)$ or 500 pg fz4 RNA $\left(\mathbf{D}, \mathbf{D}^{\prime}\right)(n=23)$. At the tailbud stage (st. 30) formation of the dorsal fin was compared to uninjected control embryos $\left(\mathbf{A}, \mathbf{A}^{\prime}\right)(n=40)$. ( $\left.\mathbf{A}^{\prime}-\mathbf{D}^{\prime}\right)$ Vibratome cross-sections of the embryos shown in $\mathbf{A}-\mathbf{D}$. White arrows indicate the dorsal fin. (E) Quantification of the experiment. (df) dorsal fin. (F) in situ hybridization for sox10 and twist mRNA in tailbud-stage embryos (st. 32) injected unilaterally with Fz4/Fz4-v1 MO or the combination of MO and fz4-v1 RNA. Asterices indicate the injected side of the embryos. (NI) non-injected side of the embryo, (wt) wild-type embryos. (G) Quantification of the CNC phenotypes. 
(Additional file 2: Figure S2M-O), supporting an involvement of Fz4-v1 in dorsal fin induction and CNC migration. Neural crest, somites and dorsal fin formation are regulated by Wnt signalling and knock-down of the Wnt co-receptor Lrp6 in Xenopus embryos leads to reduced dorsal fins, similar to Fz4-v1-depleted embryos [15].

Furthermore, it was shown that a calcium-sensitive epithelial-mesenchymal-transition (EMT) event essential for dorsal fin induction is controlled by Wnt11-R [14], which could be modulated by Fz4-v1. Our data suggest that Fz4-v1 controls Wnt signalling in the head and trunk neural crest and somites, and thereby contributes to the development of the dorsal fin.

\section{Materials and methods}

\section{Xenopus embryo manipulations}

Xenopus laevis frogs were obtained from Nasco and all experiments complied with local and international guidelines for the use of experimental animals. Xenopus eggs were obtained from females injected with 500 IU human chorionic gonadotropin (Sigma), and were fertilized in vitro. Embryos were dejellied with $2 \%$ cysteine hydrochloride $(\mathrm{pH} 8)$ and embryos were microinjected in 1x $\mathrm{MBSH}$ $(88 \mathrm{mM} \mathrm{NaCl}, 1 \mathrm{mM} \mathrm{KCl}, 2.4 \mathrm{mM} \mathrm{NaHCO} 3,0.82 \mathrm{mM}$ $\mathrm{MgSO}_{4}, 0.41 \mathrm{mM} \mathrm{CaCl}, 0.33 \mathrm{mM} \mathrm{Ca}\left(\mathrm{NO}_{3}\right)_{2}, 10 \mathrm{mM}$ HEPES (pH7.4), $10 \mu \mathrm{g} / \mathrm{ml}$ penicillin). The embryos were cultured in $0.1 \mathrm{x} \mathrm{MBSH}$ and staged according to Nieuwkoop and Faber [16].

\section{Animal cap elongation assay}

For the animal cap elongation assay, 4-cell-stage embryos were injected animally into two opposing blastomeres with synthetic RNAs. Animal caps were excised at stage 9 and cultivated in $1 \mathrm{x} \mathrm{MBSH}$ together with $10 \mathrm{ng} / \mu \mathrm{l}$ gentamycine overnight.

Synthesis of CAP-RNA and morpholinos for microinjection Capped RNAs were synthesized form linearized plasmids using the mMessage mMachine Kit (Ambion).

pCS2-Wnt3a (mouse), pCS2-Wnt8b, pCS2-Wnt11, pCS2-Fz4, pCS2-Fz4-v1, pCS2-Fz4-myc, pCS2-Fz4-v1-myc and pCS2-JNK-APF (all Xenopus) were linearized with Not1 and pSP64T-BVg1 (Xenopus) was linearized with EcoRI. Sense RNA was transcribed by SP6 polymerase.

For knock-down experiments antisense Fz4/Fz4-v1 morpholino oligonucleotide $\left(5^{\prime}\right.$-ATTATTCTTCTTCTG TTGCCGCTGA-3') or control morpholino (5' -CCTCT TACCTCAGTTACAATTTATA-3') was injected.

\section{Whole-mount in situ hybridization and Luciferase reporter assay}

Embryos were fixed in MEMFA and whole-mount in situ hybridization was performed as described [17]. pBluescriptSK-Sox10 [14] was linearized with EcoRI, and DIG-labelled antisense RNA was transcribed by T3 polymerase. pCR2.1-Twist [18] was linearized with HindIII, and DIG-labelled antisense RNA was transcribed by T7 polymerase. pCR-Blunt II-TOPO-Fz4-IntronI [9] was linearized with BamHI, and DIG-labelled antisense RNA was transcribed by T7 polymerase. Whole-mount in situ hybridization for $f z 4-v 1$ was performed using a double (5' and $3^{\prime}$ ) DIG-labelled LNA probe (5'-AGTATAGAAAG TAAACCCCCTGTG-3') from Exiqon, according to manufacturer's instructions.

For reporter assays 4-cell stage embryos were injected animally with $80 \mathrm{pg}$ M50 Super 8x Topflash [19] or $50 \mathrm{pg}$ ATF-Luciferase reporter plasmid [11] in combination with $8 \mathrm{pg}$ or $5 \mathrm{pg}$ TK-Renilla-Luciferase reporter plasmid. The reporter plasmids were injected alone or in combination with synthetic RNAs. Triplicates of $5 \mathrm{em}$ bryos were lysed according to the manufacturer's protocol (Promega) and $20 \mu \mathrm{l}$ of cell lysate was used for Luciferase detection.

\section{Additional information}

Materials and Methods used for experiments in Additional files 1 and 2: Figures S1 and S2 are provided separately in Additional file 3.

\section{Additional files}

Additional file 1: Figure S1. Fz4-v1 is a secreted protein during Xenopus development.

Additional file 2: Figure S2. Specificity of Fz4/Fz4-v1 morpholino oligonucleotide mediated knock-down of Fz4 and Fz4-v1.

Additional file 3: Materials and methods [20].

\section{Competing interests}

The authors declare that they have no competing interests.

\section{Authors' contributions}

AKG and HS designed the experiments. AKG, LTK and RKS performed the experiments and contributed to the writing of the manuscript. HS supervised the work and wrote the manuscript. All authors read and approved the final manuscript.

\section{Acknowledgements}

We thank Kirsten Linsmeier for technical support and Sarah Cramton for critically reading the manuscript. This work was supported by a research grant of the Deutsche Forschungsgemeinschaft (DFG) (STE 613/8-2).

\section{Author details}

'Section Developmental Genetics, Institute of Human Genetics, University of Heidelberg, Im Neuenheimer Feld 366, Heidelberg D-69120, Germany.

${ }^{2}$ Vascular Biology Laboratory, Institute of Life Sciences, Nalco Square, Bhubaneswar 751023, India.

Received: 27 March 2013 Accepted: 11 November 2013 Published: 19 November 2013

\section{References}

1. Jones SE, Jomary C: Secreted frizzled-related proteins: searching for relationships and patterns. Bioessays 2002, 24(9):811-820. Review.

2. Kawano Y, Kypta R: Secreted antagonists of the Wnt signalling pathway. J Cell Sci 2003, 116(Pt 13):2627-2634. Review. 
3. Bovolenta P, Esteve P, Ruiz JM, Cisneros E, Lopez-Rios J: Beyond Wnt inhibition: new functions of secreted frizzled-related proteins in development and disease. J Cell Sci 2008, 121(Pt 6):737-746. doi:10.1242/jcs.026096. Review.

4. Leyns L, Bouwmeester T, Kim SH, Piccolo S, De Robertis EM: Frzb-1 is a secreted antagonist of Wnt signaling expressed in the Spemann organizer. Cell 1997, 88(6):747-756.

5. Sagara N, Kirikoshi H, Terasaki H, Yasuhiko Y, Toda G, Shiokawa K, Katoh M: FZD4S, a splicing variant of frizzled-4, encodes a soluble-type positive regulator of the WNT signaling pathway. Biochem Biophys Res Commun 2001, 282(3):750-756.

6. Marschall Z, Fisher LW: Secreted frizzled-related protein-2 (sFRP2) augments canonical Wnt3a-induced signaling. Biochem Biophys Res Commun 2010, 400(3):299-304. doi:10.1016/j.bbrc.2010.08.043. Epub 2010 Aug 17.

7. Uren A, Reichsman F, Anest V, Taylor WG, Muraiso K, Bottaro DP, Cumberledge $S$, Rubin JS: Secreted frizzled-related protein-1 binds directly to wingless and is a biphasic modulator of Wnt signaling. J Biol Chem 2000, 275:4374-4382.

8. Mii Y, Taira M: Secreted frizzled-related proteins enhance the diffusion of Wnt ligands and expand their signalling range. Development 2009, 136(24):4083-4088. doi:10.1242/dev.032524. Epub 2009 Nov 11.

9. Swain RK, Katoh M, Medina A, Steinbeisser H: Xenopus frizzled-4S, a splicing variant of Xfz4 is a context-dependent activator and inhibitor of Wnt/beta-catenin signaling. Cell Commun Signal 2005, 3:12. doi:10.1186/1478-81 IX-3-12.

10. Janda CY, Waghray D, Levin AM, Thomas C, Garcia KC: Structural basis of Wnt recognition by frizzled. Science 2012, 337(6090):59-64. doi:10.1126/science.1222879.

11. Ohkawara B, Niehrs C: An ATF2-based luciferase reporter to monitor non-canonical Wnt signaling in Xenopus embryos. Dev Dyn 2011, 240(1):188-194.

12. Thomsen GH, Melton DA: Processed Vgl protein is an axial mesoderm inducer in Xenopus. Cell 1993, 74:433-441.

13. Tucker AS, Slack JM: Independent induction and formation of the dorsal and ventral fins in Xenopus laevis. Dev Dyn 2004, 230(3):461-467.

14. Garriock RJ, Krieg PA: Wnt11-R signaling regulates a calcium sensitive EMT event essential for dorsal fin development of Xenopus. Dev Biol 2007, 304(1):127-140.

15. Hassler C, Cruciat CM, Huang YL, Kuriyama S, Mayor R, Niehrs C: Kremen is required for neural crest induction in Xenopus and promotes LRP6-mediated Wnt signaling. Development 2007, 134(23):4255-4263.

16. Nieuwkoop and Faber: Normal table of Xenopus laevis (Daudin). New York: Garland Publishing Inc; 1994. ISBN 0-8153-1896-0.

17. Sive HL, Grainger RM, Harland RM: Early development of Xenopus laevis: a laboratory manual. Cold Spring Harbor Laboratory Press; 2000:249-274.

18. McCusker C, Cousin H, Neuner R, Alfandari D: Extracellular cleavage of cadherin- 11 by ADAM metalloproteases is essential for Xenopus cranial neural crest cell migration. Mol Biol Cell 2009, 20(1):78-89. doi:10.1091/ mbc.E08-05-0535.

19. Veeman MT, Slusarski DC, Kaykas A, Louie SH, Moon RT: Zebrafish prickle, a modulator of noncanonical Wnt/Fz signaling, regulates gastrulation movements. Curr Biol 2003, 13(8):680-685.

20. Steinbeisser H, De Robertis EM: Xenopus goosecoid: a gene expressed in the prechordal plate that has dorsalizing activity. C R Acad Sci III 1993, 316(9):959-971.

\section{Submit your next manuscript to BioMed Central and take full advantage of:}

- Convenient online submission

- Thorough peer review

- No space constraints or color figure charges

- Immediate publication on acceptance

- Inclusion in PubMed, CAS, Scopus and Google Scholar

- Research which is freely available for redistribution

Submit your manuscript at www.biomedcentral.com/submit 